\title{
Presentation rate and age effects on paired-associate recall over very brief intervals
}

\author{
MARY W. LAURENCE \\ UNIVERSITY OF TORONTO
}

Two and four second presentation rates were used to test recall of paired associates over five brief delay interval conditions in 10 year old Ss. Both presentation rate and length of delay interval had significant effects upon retention. A significant interaction showed faster loss of retention as delay interval increased at the $2 \mathrm{sec}$. rate. Twelve elderly Ss were also tested at the $2 \mathrm{sec}$. rate. Their inferior recall at very brief delay intervals is discussed in terms of initial processing difficulties.

Retention of paired associates has been shown to decrease steadily over intervals which range from 0 to $16 \mathrm{sec}$. when these are the times intervening between initial presentation of a pair and the test for recall (Peterson, Saltzman, Hillner, \& Land, 1962). These investigators, testing adult Ss, used a 2 sec. presentation rate both when stimulus and response terms were shown together and later when the stimulus term was presented alone during the test for recall. Extension of their work in two directions was the aim of the present investigation: (a) an assessment of the extent to which the diminishing retention pattern over very brief intervals demonstrated in young adults applies to the recall of both older and younger Ss, and (b) an examination of the effect of different presentation rates upon subsequent retention. In this latter connection the question of interest is whether, at a slower presentation rate than that used by Peterson et al, the presumed gains which derive from having more time to process an association will be sufficient to outweigh any disadvantage which accrues to retention levels as a result of the longer time lapse between initial presentation and test.

\section{Method}

Subjects. Two groups of 12 children each, matched for school grade level (Grades 4 and 5) and for mean age (10 years 3 months), and 12 elderly adults (mean age 74 years) served as Ss. The children were all enrolled at the Institute of Child Study school, University of Toronto. The elderly Ss were volunteers from the alumni of the University of Toronto's classes of 1914 and 1915 who had responded to a mail appeal for participants in a study of human learning. All Ss were naive with respect to paired-associate learning studies.

Lists. Twelve sets of nine words each drawn at random from a pool of 108 monosyllabic words, all of which had Thorndike-Lorge (1944) " $G$ " frequencies greater than 10 per million, served as the stimuli. A different digit from 1 to 9 was paired with each word in a list and represented the response term. The same procedure and controls in list construction and presentation which
Peterson et al used were followed here with the result that within each list measurement of retention after several different intervals of delay between initial presentation of the pair and the test recall was possible. When presentation, test and "filler" items are accounted for, each list had a total of 24 items.

The major difference between the lists constructed for this study and those of Peterson et al was that here retention was tested by presenting the stimulus alone after zero, one, two, four and eight other exposures had occurred rather than after zero, one, three, or eight other presentations. The change was designed to make the different intervals proportionally related and thereby to make comparisons between conditions easier. In each list there was one test for each of the five delay interval conditions. The position of any given condition within a list was varied randomly over the 12 lists.

Design and Procedure. Each $\mathrm{S}$ was tested on all 12 lists presented consecutively on a memory drum. One group of child Ss was tested with each word and number pair presented together for $2 \mathrm{sec}$. before the next pair was exposed. This was the presentation rate used by Peterson et al. The second, matched child group was allowed $4 \mathrm{sec}$. to study each pair as it appeared. The elderly Ss were tested at the $2 \mathrm{sec}$. rate only. Thus, at the $2 \mathrm{sec}$. rate, the delay interval between initial presentation and test for recall was $0,2,4,8$, or 16 sec. for conditions where zero, one, two, four, or eight exposures respectively intervened. At the $4 \mathrm{sec}$. presentation rate, the delay intervals were $0,4,8,16$, or $32 \mathrm{sec}$. respectively in the same comparison. Interlist interval was either 4 or $8 \mathrm{sec}$. depending upon whether $S$ was being tested at the 2 or 4 sec. presentation rate. The one exception occurred between Lists 6 and 7 when 90 sec. elapsed to permit a change of tape on the memory drum. In addition to a comparison of the effect of different presentation rates on retention under five delay interval conditions, it was possible to compare the retention levels of the child and elderly $\mathrm{S}$ both tested at the $2 \mathrm{sec}$. rate, and also to compare the performance of these groups with that of the Peterson et al Ss on three of the five conditions which overlapped in the two studies $(0,2$, and $16 \mathrm{sec}$. delay intervals).

Ss were tested individually and were asked to read aloud the word-number pair as it appeared in the window of the memory drum. When a word appeared alone, they were instructed to try to say aloud the number last seen with the word and were told to guess if necessary. The experimenter served as recorder of S's recall. 
Table 1. Mean Recall Proportions over 12 lists as a function of the Number of Items Intervening Between Initial Presentation and Test for Recall.

\begin{tabular}{lcrrrrrr} 
& & \multicolumn{7}{c}{ No. Intervening ltems } \\
Age Group & Pres. Rate & 0 & 1 & 2 & 3 & 4 & 8 \\
\hline Children & 4 sec. & .993 & .847 & .562 & .458 & .375 \\
Children & 2 sec. & 1.000 & .771 & .292 & .229 & .243 \\
Elderly & 2 sec. & .903 & .597 & .368 & & .194 & .201 \\
Peterson et al & & & & & & & \\
$\quad$ College Ss & 2 sec. & 1.000 & .760 & & .400 & & .240 \\
\hline
\end{tabular}

\section{Results and Discussion}

Table 1 reports the proportions of recall which resulted over the 12 lists for the three groups tested, and also for the Peterson et al Ss. The recall proportions are cited as a function of the number of items which intervened between initial presentation of the pair and the test for recall since this was constant regardless of presentation rate. The delay interval in terms of elapsed time may be calculated for any given group by a simple multiplication procedure: presentation rate times the number of intervening items. Table 1 discloses an obvious decline in recall as delay interval increases for all groups.

Due to the maximal performance level of some Ssespecially at the two shortest delay intervals - all retention proportions were converted to arc sines and two different analyses of variance were carried out on the transformed scores. The first of these was a comparison of the two age-matched child groups who were tested at two different presentation rates. It showed that presentation rate had a significant effect on retention level $(F=17.17, \mathrm{df}=1 / 22, \mathrm{p}<.005)$. The length of the delay interval was also overwhelmingly significant ( $F=129.18$, $\mathrm{df}=4 / 88, \mathrm{p}<.001)$ and there was a significant presentation rate by delay interval interaction $(F=3.75, d f=4 / 88$, $p<.01)$. The relationship was such that at the faster presentation rate there was significantly lower retention when two or more items intervened between presentation of the pair together and test for recall even though only half as much time had elapsed at each delay interval (4 to $16 \mathrm{sec}$. in the $2 \mathrm{sec}$. condition but 8 to $32 \mathrm{sec}$. at the $4 \mathrm{sec}$. rate). It seems probable that the faster presentation rate does not permit as adequate an initial processing of the associations as is possible at the $4 \mathrm{sec}$. rate and that retrieval difficulties and the interference potential of intervening items can become manifest, therefore, both sooner and to a more marked extent in the recall of those Ss tested at the faster rate. Presentation rate is thus shown to be an important consideration when evaluating recall levels even over very brief intervals.

When the retention levels of the elderly and the child group which was tested at the $2 \mathrm{sec}$. rate are compared, there is no significant overall difference attributable to age $(F=1.78)$. Similarity in recall is especially evident at the longer delay intervals tested. When a more restricted comparison is made at the 2 sec. delay interval where only one item intervenes between initial presentation and test for recall, there is a statistically significant difference however (the difference between mean proportional recall levels of .597 and .771 for elderly and child groups respectively yields $t=2.27$, $\mathrm{df}=11, \mathrm{p}<.05$ ). It is worthy of note too, despite a nonsignificant difference between the two groups at the 0 sec. delay interval, that four of the 12 elderly Ss failed to retain the association $100 \%$ of the time even though testing occurred immediately after presentation of a pair and no items intervened to serve as possible interference. It is especially noteworthy that one of the elderly Ss failed to retain the zero delay association in nine of the 12 instances tested.

Accordingly, it seems fair to suggest that when recall is tested after very brief or non-existent intervals under conditions of minimal or no interference, the elderly suffer greater deficit in retention than do childSs. This difference may be related to a point suggested earlierthat at a $2 \mathrm{sec}$. presentation rate, there is simply not enough time for the elderly to process the incoming associations sufficiently well to permit their becoming part of even a very short-term memory store. Such an explanatory hypothesis raises consideration of something like a "reverberating circuit" theory (Kay, 1959) to account for the nature of retentive processes which follow immediately upon perception. It further suggests that an increase in "neural noise" of sufficient magnitude to interfere with the efficient operation of the reverberating circuits may be the factor responsible for the occasional old-age breakdown in the transmission system. Clearly, what cannot get into storage in the first place cannot subsequently be retrieved even if no time lapse occurs between presentation and test for recall.

One final comparison may be made. The recall proportions of the child Ss tested at the $2 \mathrm{sec}$. rate conform very closely to those of the college freshmen in the Peterson et al study at the three delay intervals which overlap. The close correspondence between the recall levels of these two age groups has been demonstrated elsewhere for free recall learning (Laurence, in press) and the findings of the present study serve as suggestive evidence that this congruence in verbal learning behavior for two developmentally very different groups applies to paired-associate list learning as well.

\section{References}

Kay, H. Theories of learning and aging. In J. E. Birren (Ed.), Handbook of aging and the individual. Chicago: University of Chicago Press, 1959.

Laurence, Mary. Age differences in performance and subjective organization in the free recall learning of pictorial material. Canad. J. Psychol., in press.

Peterson, L. R., Saltzman, D., Hillner, K., \& Land, V. Recency and frequency in paired-associate learning. J. exp. Psychol., $1962,63,396-403$.

Thorndike, L., \& Lorge, I. The Teacher's word book of 30,000 uords. New York. Columbia University Press, 1944. 